\title{
UNIQUENESS OF SOLUTIONS TO INTEGRODIFFERENTIAL AND FUNCTIONAL INTEGRODIFFERENTIAL EQUATIONS
}

\author{
D. BAHUGUNA \\ Indian Institute of Technology \\ Department of Mathematics \\ Kanpur 208016 India \\ L.E. GAREY \\ University of New Brunswick \\ Department of Mathematics, Statistics and Computer Science \\ P.O. Box 5050, Saint John, N.B. Canada E2L $4 L 5$
}

(Received April, 1998; Revised August, 1998)

In this paper we study a class of integrodifferential and functional integrodifferential equations with infinite delay. These problems are reformulated as abstract integrodifferential and functional integrodifferential equations. We use Nagumo type conditions to establish the uniqueness of solutions to these abstract equations.

Key words: Integrodifferential Equation, Mild Solution, Nagumo Condition, Finite and Infinite Delays.

AMS subject classifications: $34 \mathrm{G} 20,24 \mathrm{~K} 15$.

\section{Introduction}

In the present work we are concerned with the following integrodifferential and functional integrodifferential equations considered in a real Banach space $X$ :

$$
\begin{gathered}
\frac{d u(t)}{d t}=f\left[t, u(t), \int_{t_{0}}^{t} k_{1}(s, u(s)) d s\right], \quad t>t_{0} \\
u\left(t_{0}\right)=x \\
\frac{d u(t)}{d t}=g\left[t, u_{t}, \int_{t_{0}}^{t} k_{2}\left(s, u_{s}\right) d s\right], \quad t>t_{0}, \\
u_{t_{0}}=\phi
\end{gathered}
$$


where in (1.1) the nonlinear map $f$ is defined from $\bar{J} \times X \times X$ into $X, J=\left(t_{0}, t_{0}+T\right]$, $\bar{J}=\left[t_{0}, t_{0}+T\right], 0<T<\infty$, the nonlinear map $k_{1}$ is defined from $\bar{J} \times X$ into $X$, $x \in X$, and in (1.2), $g$ is defined from $\bar{J} \times C_{X} \times C_{X}$ into $X, C_{X}=B U C((-\infty, 0] ; X)$ $(B U C(I ; X)$ denotes the space of bounded uniformly continuous functions from the interval $I$ into a Banach space $X$ endowed with the supremum norm), for any $u \in$ $B U C\left(\left(-\infty, t_{0}+T\right] ; X\right)$ and $t \in\left[t_{0}, t_{0}+T\right]$ the map $u_{t} \in C_{X}$ is defined by

$$
u_{t}(s)=u(t+s), \quad-\infty, s \leq 0,
$$

the nonlinear map $k_{2}$ is defined from $\bar{J} \times C_{X}$ into $C_{X}$ and $\phi \in C_{X}$.

Particular cases of (1.1) and (1.2), in which $k_{1} \equiv k_{2} \equiv 0$, have been considered by many authors, see for instance, Rogers [10], Kotta $[4,5]$. For the case $X=\boldsymbol{R}^{n}$, we refer to Kappel and Schappacher [3].

In the present work, we shall be concerned with the uniqueness of solutions only. Proving the existence of solutions to (1.1) and (1.2) will be our next concern. For the existence of solutions for the particular cases mentioned above, we refer to Hale [1], Ladas and Lakshmikantham [6], Martin and Smith [8], and Martin [9].

\section{Preliminaries}

We shall establish the uniqueness of mild solutions to (1.1) and (1.2), which would also establish the uniqueness in the case of classical solutions.

By a mild solution to (1.1) we mean a continuous function $u \in C(\bar{J}, X)$ such that

$$
u(t)=x+\int_{t_{0}}^{t} f\left[s, u(s), \int_{t_{0}}^{s} k_{1}(\tau, u(\tau)) d \tau\right] d s, \quad t_{0} \leq t \leq t_{0}+T,
$$

where $x \in X$. By a mild solution to (1.2) we mean a continuous function $u \in$ $B U C\left(\left(-\infty, t_{0}+T\right], X\right)$ such that

$$
u(t)=\left\{\begin{array}{cc}
\phi\left(t-t_{0}\right), & -\infty \leq t \leq t_{0}, \\
\phi(0)+\int_{t_{0}}^{t} f\left[s, u_{s}, \int_{t_{0}}^{s} k_{1}\left(\tau, u_{\tau}\right) d \tau\right] d s, & t_{0} \leq t \leq t_{0}+T,
\end{array}\right.
$$

where $\phi \in C_{X}$.

We consider a function $h \in C\left(J, \boldsymbol{R}^{+}\right)$satisfying the following condition

(H)

For, instance, we may take

$$
\lim _{t \rightarrow t_{0}^{+}} \int h(t) d t=-\infty
$$

$$
h(t)=\frac{1}{\left(t-t_{0}\right)^{2}} \quad t \in J .
$$

Then $h$ satisfies condition (H). 
Remark: If $h \in C\left(J, \boldsymbol{R}^{+}\right)$satisfies condition (H), then the function $\tilde{h} \in$ $C\left(J, \boldsymbol{R}^{+}\right)$given by

$$
\tilde{h}(t)=h(t)+C, \quad t \in J
$$

for any positive constant $C$ also satisfies condition (H).

The main tool for proving the uniqueness of solutions is the following lemma due to Kotta [5]. This result is a generalization of an analogous result of Rogers [10]. For the sake of completeness, we shall give a proof of the lemma here.

Lemma 2.1: Let $u \in C\left(\bar{J}, \boldsymbol{R}^{+}\right)$and suppose that

(i) $u(t) \leq \int_{t_{0}}^{t} h(s) u(s) d s$,

(ii) $u(t)=o\left(e^{\int h(t) d t}\right)$, as $t \rightarrow t_{0}^{+}$,

where $h \in C\left(J, \boldsymbol{R}^{+}\right)$satisfying condition (H). Then $u \equiv 0$ on $\bar{J}$.

Proof: Let

Using $(i)$ and (2.3) we obtain

$$
F(t)=\int_{t_{0}}^{t} h(s) u(s) d s, \quad t \in J .
$$

$$
\frac{d}{d t} F(t)=h(t) u(t) \leq h(t) F(t) .
$$

Therefore,

$$
\frac{d}{d t}\left(e^{-\int h(t) d t} F(t)\right) \leq 0 .
$$

Inequality (2.4) implies that $F(t) e^{-\int h(t) d t}$ is a nonincreasing function on $J$.

From (ii) it follows that for every $\epsilon>0$, there exists a $\delta>0$ such that for $t \in J$ with $0<t-t_{0}<\delta$, we have

Now,

$$
\begin{gathered}
e^{-\int h(t) d t} F(t)=e^{-\int h(t) d t} \int_{t_{0}}^{t} h(s) u(s) d s \\
\leq \epsilon e^{-\int h(t) d t} \int_{t_{0}}^{t} h(s) e^{\int h(s) d s} d s .
\end{gathered}
$$

$$
\frac{d}{d t} e^{\int h(t) d t}=h(t) e^{\int h(t) d t}
$$

Integrating (2.6) over the interval $\left(t_{0}, t\right)$, we get

$$
\left.e^{\int h(t) d t}\right|_{t_{0}} ^{t}=\int_{t_{0}}^{t} h(s) e^{\int h(s) d s} d s .
$$

Using condition (H) in (2.7), we get 


$$
e^{\int h(t) d t}=\int_{t_{0}}^{t} h(s) e^{\int h(s) d s} d s
$$

Using (2.8) in (2.5), we get

$$
e^{-\int h(t) d t} F(t) \leq \epsilon
$$

From (2.9), we have that

$$
\lim _{t \rightarrow t_{0}^{+}} e^{-\int h(t) d t} F(t)=0 .
$$

Hence, $F(t) \equiv 0$ on $\bar{J}$, which in turn implies that $u \equiv 0$ on $\bar{J}$.

\section{Main Result}

We first state and prove the following uniqueness theorem for (1.1).

Theorem 3.1: Suppose that $f: \bar{J} \times X \times X \rightarrow X$ is a continuous function and satisfies the conditions

$$
\left\|f\left(t, x_{1}, y_{1}\right)-f\left(t, x_{2}, y_{2}\right)\right\| \leq h(t)\left[\left\|x_{1}-x_{2}\right\|+\left\|y_{1}-y_{2}\right\|\right]
$$

for any $t \in \bar{J}, x_{i}, y_{i} \in X, i=1,2 ;$ and

$$
\|f(t, x(t), y(t))\|=o\left(h(t) e^{\int h(t) d t}\right) \text { as } t \rightarrow t_{0}^{+},
$$

for any $t \in \bar{J}, x, y \in C(\bar{J} ; X)$, where $h \in C\left(J, \boldsymbol{R}^{+}\right)$satisfies condition (H). Further, suppose that $k_{1}: \bar{J} \times X \rightarrow X$ satisfies

$$
\left\|k_{1}\left(t, x_{1}\right)-k_{1}\left(t, x_{2}\right)\right\| \leq C(t)\left\|x_{1}-x_{2}\right\|
$$

for any $t \in \bar{J}, x_{i} \in X, i=1,2$; where $C(t)$ is a nonnegative integrable function on $J$. Then (1.1) has at most one solution.

Proof: Let $x(t)$ and $y(t)$ be solutions of (1.1). Then we have

$$
\begin{aligned}
\|x(t)-y(t)\| \leq \int_{t_{0}}^{t} \| f\left[\left(s, x(s), \int_{t_{0}}^{s} k_{1}(\tau, x(\tau)) d \tau\right]\right. \\
\quad-f\left[\left(s, y(s), \int_{t_{0}}^{s} k_{1}(\tau, y(\tau)) d \tau\right] \| d s .\right.
\end{aligned}
$$

Using (3.1) and (3.3) in (3.4), we get

$$
\|x(t)-y(t)\| \leq \int_{t_{0}}^{t} h(s)\left[\|x(s)-y(s)\|+\int_{t_{0}}^{s}\left\|k_{1}(\tau, x(\tau))-k_{1}(\tau, y(\tau))\right\| d \tau\right] d s
$$




$$
\begin{gathered}
\leq \int_{t_{0}}^{t} h(s)\left[\|x(s)-y(s)\|+\int_{t_{0}}^{s} C(\tau)\|x(\tau)-y(\tau)\| d \tau\right] d s \\
\leq \int_{t_{0}}^{t}\left(h(s)+C_{T}\right)\left\{\sup _{t_{0} \leq \tau \leq s}\|x(\tau)-y(\tau)\|\right\} d s \\
\leq \int_{t_{0}}^{t} \tilde{h}(s)\left\{\sup _{t_{0} \leq \tau \leq s}\|x(\tau)-y(\tau)\|\right\} d s
\end{gathered}
$$

where

and

$$
C_{T}=\int_{t_{0}}^{t_{0}+T} C(s) d s
$$

$$
\tilde{h}(t)=h(t)+C_{T}
$$

Now, (3.5) implies that for every $t_{0} \leq \eta \leq t$, we have

$$
\begin{aligned}
\| x(\eta) & -y(\eta) \| \leq \int_{t_{0}}^{\eta} \tilde{h}(s)\left\{\sup _{t_{0} \leq \tau \leq s}\|x(\tau)-y(t)\|\right\} d s \\
& \leq \int_{t_{0}}^{t} \tilde{h}(s)\left\{\sup _{t_{0} \leq \tau \leq s}\|x(\tau)-y(\tau)\|\right\} d s .
\end{aligned}
$$

From (3.6) we get

$$
\sup _{t_{0} \leq \eta \leq t}\|x(\eta)-y(\eta)\| \leq \int_{t_{0}}^{t} \tilde{h}(s)\left\{\sup _{t_{0} \leq \tau \leq s}\|x(\tau)-y(\tau)\|\right\} d s .
$$

Replacing the dummy variable $\eta$ on the left-hand side of inequality (3.7) by $\tau$, we finally get

$$
\sup _{t_{0} \leq \tau \leq t}\|x(\tau)-y(\tau)\| \leq \int_{t_{0}}^{t} \tilde{h}(s)\left\{\sup _{t_{0} \leq \tau \leq s}\|x(\tau)-Y(\tau)\|\right\} d s .
$$

Now, using (3.2) in inequality (3.4) we have that for $\epsilon>0$, there exists $\delta>0$ such that for $0<t-t_{0}<\delta$,

$$
\begin{gathered}
\|x(t)-y(t)\| \leq \epsilon \int_{t_{0}}^{t} h(s) e^{\int h(s) d s} d s \\
\leq \epsilon \int_{t_{0}}^{t}\left(h(s)+C_{T}\right) e^{C_{T^{s}}} e^{\int h(s) d s} d s
\end{gathered}
$$




$$
=\epsilon \int_{t_{0}}^{t} \tilde{h}(s) e^{\int \tilde{h}(s) d s} d s
$$

Again, for $t_{0} \leq \tau \leq t$, we have

$$
\begin{gathered}
\|x(\tau)-y(\tau)\| \leq \epsilon \int_{t_{0}}^{\tau} \tilde{h}(s) e^{\int \tilde{h}(s) d s} d s \\
\leq \epsilon \int_{t_{0}}^{t} \tilde{h}(s) e^{\int \tilde{h}(s) d s} d s .
\end{gathered}
$$

Taking supremum in $(3.10)$ we get

$$
\sup _{t_{0} \leq \tau \leq t}\|x(\tau)-y(\tau)\| \leq \epsilon \int_{t_{0}}^{t} \tilde{h}(s) e^{\int \tilde{h}(s) d s} d s=\epsilon e^{\int \tilde{h}(t) d t} .
$$

We obtained the desired result using Lemma 2.1. This completes the proof of Theorem 3.1.

Next, we state and prove below a similar uniqueness result for (1.2).

Theorem 3.2: Suppose that $g: \bar{J} \times C_{X} \times C_{X} \rightarrow C_{X}$ is continuous and satisfies the condition

$$
\left\|g\left(t, \phi_{1}, \psi_{1}\right)-g\left(t, \phi_{2}, \psi_{2}\right)\right\|_{C_{X}} \leq h(t)\left[\left\|\phi_{1}-\phi_{2}\right\|_{C_{X}}+\left\|\psi_{1}-\psi_{2}\right\|_{C_{X}}\right]
$$

for any $t \in \bar{J}, \phi_{i}, \psi_{i} \in C_{X}, i=1,2$; and

$$
\left\|g\left(t, \phi_{t}, \psi_{t}\right)\right\|_{C_{X}}=o\left(h(t) e^{\int h(t) d t}\right), \text { as } t \rightarrow t_{0}^{+},
$$

for any $\left.t \in \bar{J}, \phi, \psi \in C\left(-\infty, t_{0}+T\right] ; X\right)$, where $h \in C\left(J, \boldsymbol{R}^{+}\right)$satisfies condition (H). Suppose that $k_{2}: \bar{J} \times C_{X} \rightarrow C_{X}$ is continuous and satisfies

$$
\left\|k_{2}\left(t, \phi_{1}\right)-k_{2}\left(t, \phi_{2}\right)\right\|_{C_{X}} \leq D(t)\left\|\phi_{1}-\phi_{2}\right\|_{C_{X}}
$$

where $D(t)$ is a nonnegative integrable function on $J$. Then (1.2) has at most one solution.

Proof: Suppose that $x$ and $y$ are two solutions to (1.2). Then $x_{t_{0}}=y_{t_{0}}=\phi$ implies that

$$
x \equiv y, \text { on }\left(-\infty, t_{0}\right]
$$

Therefore,

$$
\left.\left\|x_{t}-y_{t}\right\|_{C_{X}}=\sup _{s \in(-\infty, 0]} \| x(t+s)-y t+s\right)\left\|=\max _{\theta \in\left[t_{0}, t\right]}\right\| x(\theta)-y(t) \|
$$

From definition (2.2) of mild solutions to (1.2) and (3.15) we have 


$$
\left\|x_{t}-y_{t}\right\|_{C_{X}} \leq \int_{t_{0}}^{t}\left\|g\left[s, x_{s}, \int_{t_{0}}^{s} k_{2}\left(\tau, x_{\tau}\right) d t\right]-g\left[s, y_{s} \int_{t_{0}}^{s} k_{2}\left(\tau, y_{\tau}\right) d \tau\right]\right\|_{C_{X}} d s
$$

Using (3.12) and (3.14) in (3.16), we obtain

$$
\begin{gathered}
\left\|x_{t}-y_{t}\right\|_{C_{X}} \leq \int_{t_{0}}^{t} h(s)\left[\left\|x_{s}-y_{s}\right\|_{C_{X}}+\int_{t_{0}}^{s} D(\tau)\left\|x_{\tau}-y_{\tau}\right\|_{C_{X}} d \tau\right] d s \\
\leq \int_{t_{0}}^{t}\left(h(s)+\int_{t_{0}}^{s} D(\tau) d \tau\right)\left[\left\|x_{s}-y_{s}\right\|_{C_{X}} d s\right. \\
\leq \int_{t_{0}}^{t}\left(h(s)+D_{T}\right)\left\|x_{s}-y_{s}\right\|_{C_{X}} d s \\
\leq \int_{t_{0}}^{t} \tilde{h}(s)\left\|x_{s}-y_{s}\right\|_{C_{X}} d s
\end{gathered}
$$

where

and

$$
D_{T}=\int_{t_{0}}^{t_{0}+T} D(s) d s
$$

$$
\tilde{h}(t)=h(t)+D_{T}, \quad t \in J
$$

Now, using (3.13) in (3.16), we have that for $\epsilon>0$, there exists $\delta>0$ such that for $0<t-t_{0}<\delta$

$$
\begin{gathered}
\left\|x_{t}-y_{t}\right\|_{C_{X}} \leq \epsilon \int_{t_{0}}^{t} h(s) e^{\int h(s) d s} d s \\
\leq \epsilon \int_{t_{0}}^{t}\left(h(s)+D_{T}\right) e^{D_{T^{s}} e^{\int h(s) d s} d s} \\
\leq \epsilon \int_{t_{0}}^{t} \tilde{h}(s) e^{\int \widetilde{h}(s) d s} d s \\
=\epsilon e^{\int \tilde{h}(t) d t} .
\end{gathered}
$$

Again, we apply Lemma 2.1 to get the desired result. This completes the proof of Theorem 3.2. 


\section{Acknowledgement}

The first author would like to acknowledge the financial support provided by the National Board for Higher Mathematics under its research project No. 48/2/97-R\&DII/2003 to carry out this work.

\section{References}

[1] Hale, J.K., Theory of Functional Differential Equations, Springer-Verlag, New York 1977.

[2] Hale, J.K. and Kato, T., Phase space for retarded equations with infinite delay, Funkcial. Ekvac. 21 (1978), 11-44.

[3] Kappel, F. and Schappacher, W., Some considerations to the fundamental theory of infinite delay equations, J. Diff. Eqns. 37 (1980), 141-183.

[4] Kotta, E.S., Uniqueness of solutions of functional differential equations in Hilbert spaces, J. Inst. Math. Comp. Sci. (Math Ser.) 7 (1994), 115-119.

[5] Kotta, E.S., On the uniqueness of solutions for ordinary and functional differential equations, J. Inst. Math. Comp. Sci. (Math. Ser.) 8 (1995), 129-133.

[6] Ladas, G.E. and Lakshmikantham, V., Differential Equations in Abstract Spaces, Academic Press, New York 1972.

[7] Lakshmikantham, V., Leela, S. and Moauro, V., Existence and uniqueness of solutions of delay differential equations on closed subsets on a Banach space, Nonlinear Anal. 2 (1978), 311-327.

[8] Martin, R.H. and Smith, H.L., Abstract functional differential equations and reaction-diffusion systems, Trans. Amer. Math. Soc. 321 (1990), 1-44.

[9] Martin, R.H., Nonlinear Operators and Differential Equations in Banach Spaces, Wiley, New York 1976.

[10] Rogers, T., On Nagumo's condition, Canad. Math. Bull. 15 (1972), 609-611. 


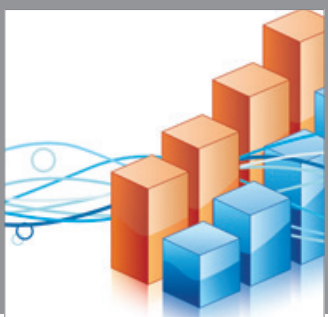

Advances in

Operations Research

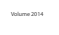

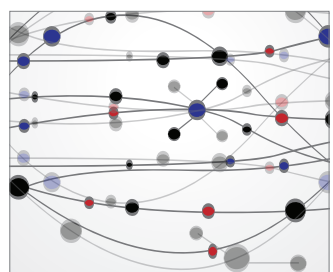

\section{The Scientific} World Journal


International Journal of

Mathematics and

Mathematical

Sciences
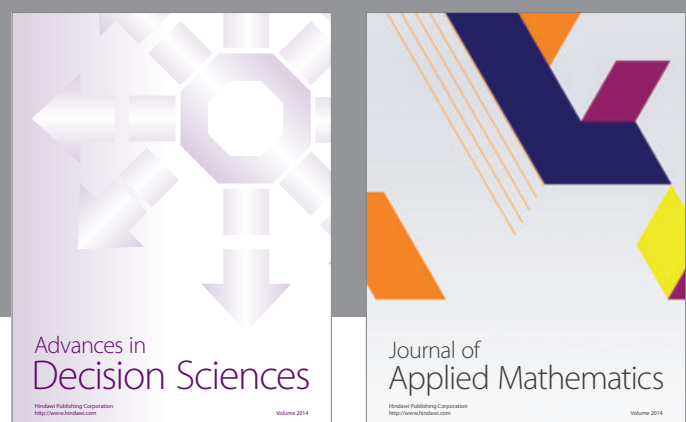

Journal of

Applied Mathematics
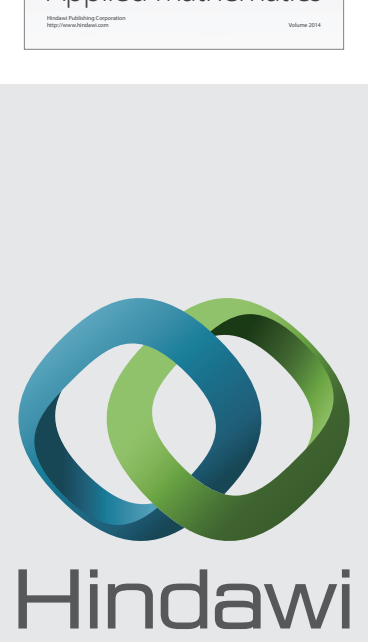

Submit your manuscripts at http://www.hindawi.com
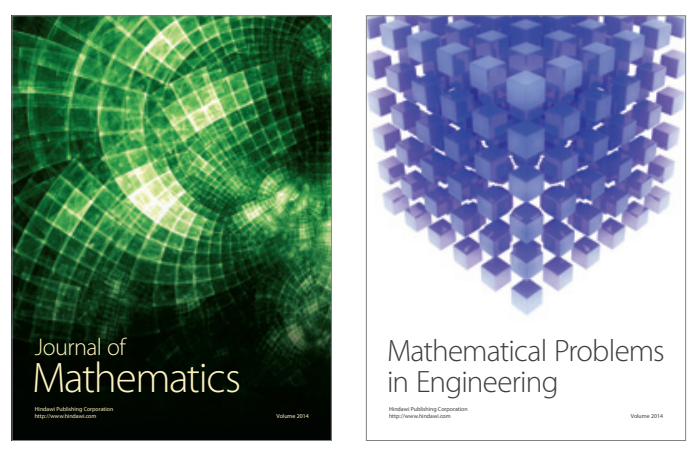

Mathematical Problems in Engineering


Journal of

Function Spaces
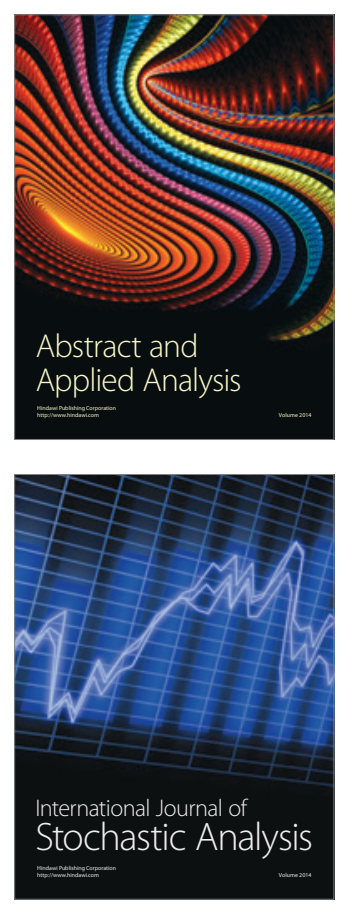

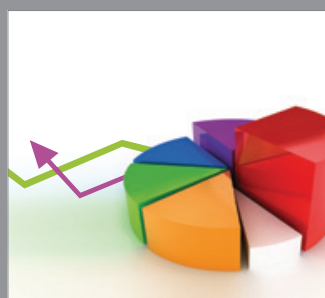

ournal of

Probability and Statistics

Promensencen
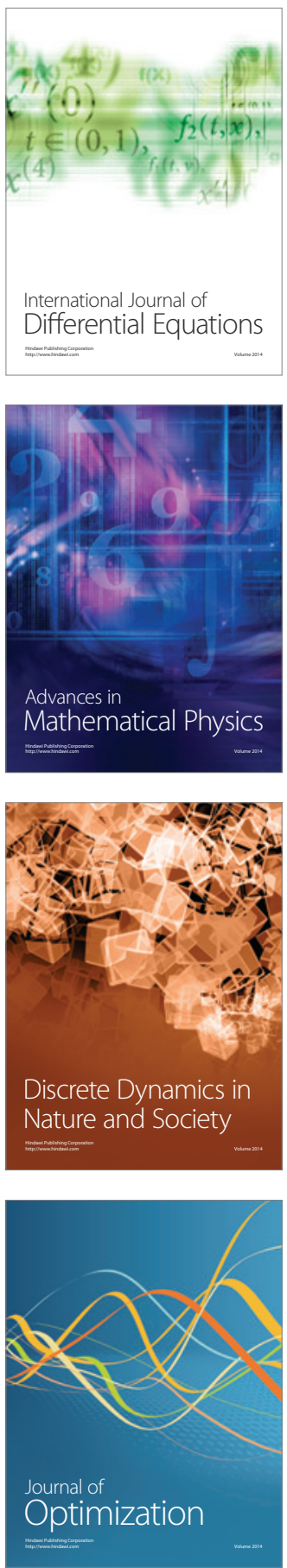